\title{
Interactions between fecal bacteria, bile acids and components of tomato pomace
}

\author{
Krzysztof Dziedzic $^{1,2}$ (D) Danuta Górecka ${ }^{3} \cdot$ Artur Szwengiel $^{1} \cdot$ Jan Michniewicz $^{1} \cdot$ \\ Agnieszka Drożdżyńska $^{4} \cdot$ Jarosław Walkowiak $^{2}$
}

Received: 18 April 2018/Revised: 12 November 2018/Accepted: 28 November 2018/Published online: 8 December 2018

(C) The Author(s) 2018

\begin{abstract}
The tomato pomace obtained during processing as a residue of tomato processing from large industry. The interactions between tomato pomace and fecal bacteria, bile acids during in vitro digestion were studied. Digestion was carried out by using bioreactor in anaerobic conditions. Tomato pomace can significantly affect the count of fecal bacteria and the solubility of bile acids in in vitro digestion due to bonding ability of their proteins/peptides. The availability and use of bile acids does not only depend on the interactions between bile acids and bacteria, but also the interactions of bile acids with digested food components. Tomato pomace characterized high dietary fiber content and its fractions: $17.64-21.53 \%$ for cellulose and $13.48-18.63 \%$ for lignin. Given our results we supposed that fecal bacteria can use primary bile acids, as their source of energy in an environment where carbon availability is limited.
\end{abstract}

Keywords Cholic acid · Deoxycholic acid · In vitro digestion $\cdot$ Lithocholic acid $\cdot$ Tomato waste

Krzysztof Dziedzic

krzysztof.dziedzic@up.poznan.pl

1 Institute of Food Technology and Plant Origin, Poznan University of Life Sciences, Wojska Polskiego 31, 60-624 Poznan, Poland

2 Department of Gastroenterology and Metabolic Diseases, Poznan University of Medical Sciences, Szpitalna 27/33, 60-572 Poznan, Poland

3 Department of Gastronomy Science and Functional Food, Poznan University of Life Sciences, Wojska Polskiego 31, 60-624 Poznan, Poland

4 Department of Biotechnology and Food Microbiology, Poznan University of Life Sciences, Wojska Polskiego 48, 60-627 Poznan, Poland

\section{Introduction}

Tomato pomace (TP) is the most abundant waste in fruit and vegetable industries (11 million tone of tomato waste including 4 million tone of tomato pomace). The chemical composition of TP varies depending on the agricultural procedures applied and technical processing (Bajerska et al., 2015). This is a rich source of some nutritive compounds, such as: protein and lysine (15-24\%), fat (5-20\%, mainly linoleic acid), total sugar $(28-51 \%)$ and mineral substances (3-6\%) - all figures given in dry basis. They can be an excellent source of bioactive substances used as additives in food production (Güneşer et al., 2015; Stinco et al., 2016). TP is a good source of dietary fibre (mainly cellulose, hemicellulose, and lignin) (Borguini and Ferraz Da Silva Torres, 2009). The waste products of food production still contain very large amounts of secondary metabolites-primarily polyphenolic substances likes flavanones (naringenin, glycosilated derivatives), flavonols (quercetin, rutin, and kaempferol) and carotenoids, such as lycopene (Bajerska et al., 2015; Böhm, 2012; Chen et al., 2013; Kun et al., 2006; Stinco et al., 2016). There have been several attempts to enrich food products by using pomace as a source of dietary fibre and other bioactive compounds. Dry tomato peel was used during production of meat products (Previtera et al., 2016), snacks (DehghanShoar et al., 2010; Dehghan-Shoar et al., 2011), rye bread (Bajerska et al., 2015), and tomato pasta (Reboul et al., 2005). The physiological activity of food components such as dietary fibre, protein and phytosterols in reducing plasma cholesterol has been extensively reported. Various freeze dried tomato products such as powder, pomace, juice, and other products have been reported to lower plasma cholesterol in hamsters and humans. Several mechanism of lowering LDL (low density lipoprotein) 
fraction cholesterol level have been documented. Changes in the levels of plasma cholesterol, fecal cholesterol and intestinal cholesterol absorption have been discussed (Shao et al., 2013). TP can also be an excellent source for the production of some prebiotics because of its nutritive value for microbial growth (Del Valle et al., 2006). In literature, there is a lack of evidence of the role of food products enriched with dietary fibre fractions obtained from tomato pomace in stimulation or retardation of fecal bacteria growth, and the ability of fecal bacteria in the management of bile salts and bile acids (Dziedzic et al., 2016). Begley et al. (2005) described that E. coli is considered to be very bile resistant and can be isolate from gallbladder and bile of humans. E. coli growth was observed in duodenum of a gastrointestinal model in the presence of very high concentration of bile, while Gram-positive bacteria in the same environment were deactivated. They showed also that bile tolerance is strain-specific. The effect of bile tolerance on a collection of 38 Lactobacillus strains were described: 5 strains were tolerant to bile of most than $0.3 \%$ concentration, whilst the growth of other strains was delayed. Among Bifidobacterium strains, B. infantis had the best survival rate but B. longum had the lowest (Begley et al., 2005).

Novelty of this experiment is investigation of few factors, which can be responsible for lipids manage in in vitro model. Therefore the aim of this study was to evaluate the role of TP in the conditioning of fecal bacteria and their ability to bind primary and secondary bile acids.

\section{Materials and methods}

\section{Materials}

TP used for this research was obtained from a Polish Food Industry Company "HJH Polska Sp. z. o. o." (Grandimat variety, ripe and ready for consumption, from harvests in 2013 (TP1) and 2014 (TP2). TP was dried using a lyophilizer, and then kept at room temperature in the dark. Before the analysis, the sample was ground in a Foss Tecator mill (Hillerod, Sweden). Following reagents were used: pepsin, pancreatin, bile acids (cholic, deoxycholic and lithocholic acid), sodium bicarbonate, acetic acid, propionic acid, lactic acid, butyric acid purchased from Sigma-Aldrich (Seelze, Germany); hydrochloric acid, sodium hydroxide, ethanol, acetone, neutral disodium versenate dehydrate, disodium tetraborate decahydrate, disodium hydrogen phosphate, ethylene glycol, sulfuric acid (Poch, Gliwice, Poland); $N$-cetyl- $N, N, N$-trimethylammoniumbromid, Kanamycine Esculine Azide Agar, TOS (transoligosacharide) propionate agar medium with MUP (Lithium Mupirocin), Endo agar, and MRS agar. (Merck,
Darmstadt, Germany); thermostable $\alpha$-amylase (Novozymes, Bagsvaerd, Denmark).

\section{In vitro digestion}

The digestive process (30 $\mathrm{g}$ of sample) was carried out according to Dziedzic et al. $(2015 ; 2016)$ The size of the reaction tank was modified $(1 \mathrm{~L})$. The environment of the stomach, small and large intestine was reproduced as closely as possible (Ulleberg et al., 2011) with its $\mathrm{pH}$ and enzymes i.e. pepsin $(0.576 \mathrm{~g}$ in $12 \mathrm{~mL}$ of $0.1 \mathrm{M}$ sterilized hydrochloric acid) - the first stage of digestion, pancreatin (0.12 g), and bile acids (cholic acid, CA; deoxycholic acid, DCA; lithocholic acid, LHA, each $0.36 \mathrm{~g}$ )—mixed together in $30 \mathrm{~mL}$ of $0.1 \mathrm{M}$ sterilized sodium bicarbonate as a suspension - the second stage of digestion. Subsequently, a mix of fecal bacteria, previously isolated from a healthy 24 year old male, was added at stage 2 (after $30 \mathrm{~min}$., anaerobic conditions), in the amount of $10^{4}-10^{6} \mathrm{CFU} / \mathrm{mL}$. Next after $2 \mathrm{~h}$ the $\mathrm{pH}$ was changed again for 8.0- the third stage of digestion (18 h, large intestine). A bioreactor (300 mL of total volume, Sartorius Stedim, Biostat B Plus, Goettingen, Germany) was used as the in vitro digestive tract, and samples were obtained from three stages of digestion (1-duodenum, $\mathrm{pH} 6.0 ; 2$-ileum, $\mathrm{pH} 7.2 ; 3-$ colon, $\mathrm{pH}$ 8.0). The simulation of the gastrointestinal tract was conducted at $37{ }^{\circ} \mathrm{C}$, in anaerobic conditions and at the stirring speed of $200 \mathrm{rpm}$.

\section{Dietary fiber assay}

\section{Total dietary fiber (TDF)}

The content of TDF, soluble dietary fiber (SDF) and insoluble dietary fiber (IDF) was analyzed using the enzymatic method (Dziedzic et al., 2016). The following enzymes were used: thermostable $\alpha$-amylase (Termamyl $120 \mathrm{~L}, \mathrm{pH} 6.0,90^{\circ} \mathrm{C}, 15 \mathrm{~min}$.); pepsin ( $\mathrm{pH} 1.5,40{ }^{\circ} \mathrm{C}$, $1 \mathrm{~h})$, and pancreatin ( $\left.\mathrm{pH} 6.8,40{ }^{\circ} \mathrm{C}, 1 \mathrm{~h}\right)$. Analyses were performed using a Fibertec System 1023 apparatus (Foss, Hillerod, Sweden).

\section{Detergent fiber determination}

The content of neutral dietary fiber (NDF), consisting of acid detergent fiber (ADF) and acid detergent lignin (L), was determined using the detergent method, previously used by Dziedzic et al. (2016). Thermostable $\alpha$-amylase was used to digest starch. The reagents applied to estimate the content of neutral detergent fiber (NDF) were: neutral disodium versenate dehydrate, disodium tetraborate decahydrate, disodium hydrogen phosphate, ethylene glycol and redistilled water. The reagents used to estimate the 
content of ADF were: sulfuric acid (1 N, Poch, Gliwice, Poland, pure p.a.) and $N$-cetyl- $N, N, N$-trimethylammoniumbromid. The reagent used to estimate the content of $\mathrm{L}$ was sulfuric acid $(72 \%)$. Hemicellulose $(\mathrm{H})$ content was calculated from the difference between NDF and ADF. Cellulose (C) content was calculated from the difference between ADF and L. Analyses were conducted using a Fibertec System M 1020 apparatus by Tecator (Foss, Hillerod, Sweden).

\section{Bile acid assay}

Bile acids (CA, DCA and LHA) were analyzed using LCMS method described by Dziedzic et al. (2015; 2016). Ultra high-performance liquid chromatography electrospray ionization mass spectrometry analysis was performed using a DionexUltiMate 3000 UHPLC (Thermo Fisher scientific, Sunnyvale, CA, USA) coupled with a Bruker maXis impact ultrahigh resolution orthogonal quadrupoletime-of-light accelerator (qTOF) equipped with an ESI source and operated in the positive-ion Dean distance measure.

\section{Fecal bacteria identification (CFU)}

Microbiological research was carried out according to international standards (Wohlsen et al., 2006). The count of Enterococcus spp., Bifidobacterium spp., E. coli and Lactobacillus spp. in the experimental samples were determined using the general pour plate technique on Kanamycine Esculine Azide Agar for Enterococcus spp., TOS agar with MUP Selective Supplement for Bifidobacterium spp., Endo agar for E. coli, and MRS agar for Lactobacillus spp.

\section{Short chain fatty acid assay (SCFA)}

Determination of organic acids (acetic acid, propionic acid, lactic acid, butyric acid) was carried out using a UHPLC (VWR-HITACHI LaChrom Elite) system consisting of an autosampler (model L-2200), pump (model L-2130) and a UV detector ( $L-2400)$ connected in a series (Primec et al., 2017). Analyses were performed isocratically at a flow rate of $0.6 \mathrm{~mL} / \mathrm{min}$ at $40{ }^{\circ} \mathrm{C}$, on Rezex ROA-Organic Acid $\mathrm{H}+, \quad 300 \times 7.8 \mathrm{~mm}$ (Phenomenex) column. Standards (lactic acid-1.1, 0.55, 0.275, 0.11 g/L; acetic acid-1.0, 0.5, 0.25, 0.1 g/L; propionic acid-1.0, 0.5, 0.25, 0.1 g/L, and butyric acid- $0.55,0.275,0.1375,0.055 \mathrm{~g} / \mathrm{L}$ ) were used.

\section{Statistical analysis}

The experiments were executed in three independent trials. $T$ test and Hierarchical cluster analysis were carried out. Tree plots were scaled to a standardized scale (dlink/ dmax * 100). Non-hierarchical cluster analysis (k-means clustering) was performed to form a grouping of control/tomato pomace (TP1 and TP2) samples at the three stages of digestion (pH: 6.0, 7.2 and 8.0). Principal component analysis (PCA) technique was used to reduce the dimensionality of data and to present the samples in a new coordinate system. Statistica software, Version 10, StatSoft Inc. (Tulsa, OK, USA) was used to carry out statistical analysis.

\section{Results and discussion}

Fat, protein, ash content, dietary fiber, and its soluble and insoluble fractions were estimated. Results of t-test for independent samples show significant differences between analyzed samples. The differences between TP1 and TP2 showed that year of harvest had influence for content of investigated substances in the samples, Table 1. TP1 had the highest content of fat, NDF, $\mathrm{H}$ and $\mathrm{L}$ in comparison to TP2.

The in vitro digestion was carried out in three stages, where $\mathrm{pH}$ was $6.0,7.2$, and 8.0, respectively. The control sample consisted of a mixture of reagents and bacteria without TP. During in vitro digestion the concentration of individual bile salts, SCFA, and CFU were determined at each stage, Table 2. First, the exploration of two-

Table 1 Characteristics of the tomato pomaces obtained in two consecutive years (TP1 and TP2), SD-the standard deviation (g/ $100 \mathrm{~g}$ of product)

\begin{tabular}{lrr}
\hline Component & \multicolumn{1}{c}{ TP1* \pm SD } & \multicolumn{1}{c}{ TP2 $2^{*} \pm$ SD } \\
\hline Fat & $9.72^{\mathrm{b}} \pm 0.50$ & $7.23^{\mathrm{a}} \pm 0.12$ \\
Protein & $18.18^{\mathrm{a}} \pm 0.13$ & $18.93^{\mathrm{b}} \pm 0.21$ \\
Ash & $4.01^{\mathrm{a}} \pm 0.02$ & $4.14^{\mathrm{b}} \pm 0.05$ \\
NDF & $49.33^{\mathrm{a}} \pm 0.34$ & $45.97^{\mathrm{b}} \pm 0.39$ \\
Cellulose & $17.64^{\mathrm{a}} \pm 0.12$ & $21.53^{\mathrm{b}} \pm 0.56$ \\
Hemicellulose & $13.01^{\mathrm{b}} \pm 0.87$ & $10.96^{\mathrm{a}} \pm 0.69$ \\
Lignin & $18.69^{\mathrm{b}} \pm 0.67$ & $13.48^{\mathrm{a}} \pm 0.18$ \\
SDF & $0.61^{\mathrm{a}} \pm 0.08$ & $0.87^{\mathrm{b}} \pm 0.06$ \\
IDF & $45.27^{\mathrm{a}} \pm 0.56$ & $49.16^{\mathrm{b}} \pm 0.57$ \\
TDF & $45.88^{\mathrm{a}} \pm 0.62$ & $50.03^{\mathrm{b}} \pm 0.63$ \\
\hline
\end{tabular}

IDF, insoluble dietary fiber; NDF, neutral detergent fiber; SDF, soluble dietary fiber; TDF, total dietary fiber

* Superscripts indicate significant difference in rows between means $(p<0.05)$ 


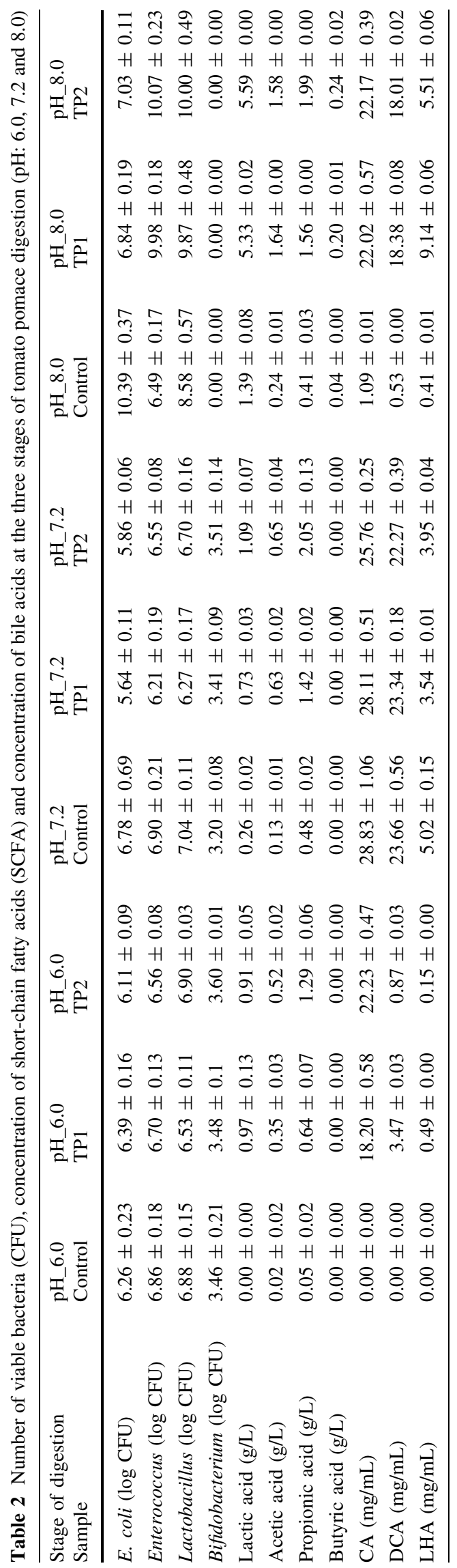


dimensional data matrix using cluster analysis was conducted (Tables 1, 2, Fig. 1).

The analysis of hierarchical tree showed 2 different groups of results (the height of the cut-off point $80 \%$ ). The first cluster consists of control samples of high homogeneity, while the second cluster contains TP samples. We can conclude that TP samples had an effected on the fecal bacteria and the profile of analyzed substances during in vitro digestion (Table 2). It is possible to distinguish three subgroups of six elements each, which are specific to further stages of in vitro digestion (the height of the cut-off point $6 \%$ ). However, the most diversified group consisted of samples obtained from the third stage of digestion $(\mathrm{pH}$ 8.0). At the second stage, k-means clustering was conducted, assuming a priori the number of groups equal to 3 based on the results of cluster analysis. The results of k-means analysis confirm the earlier observations as to the assignment of the samples to three groups. The evaluation of discriminant power of variables $(p<0.05)$ by using ANOVA method proves that only the concentration of DCA does not differ significantly within the analyzed groups (Table 3).

To determine the relationships between variables, variables-samples, and samples alone, a PCA analysis was performed. The results were presented in Fig. 2 in a system of two principal components (PCs), explaining $80.41 \%$ of total variance. In this way the number of original dimensions was reduced from 13 original variables to 2 newly created (PC 1 and PC 2). Instances excluding control samples were examined, because they exhibited significant differences to TP samples, as demonstrated above. Solubility of bile acids in control samples was lower than in the case of TP samples, what is related to absence of short chain fatty acids (Table 2). A positive correlation was

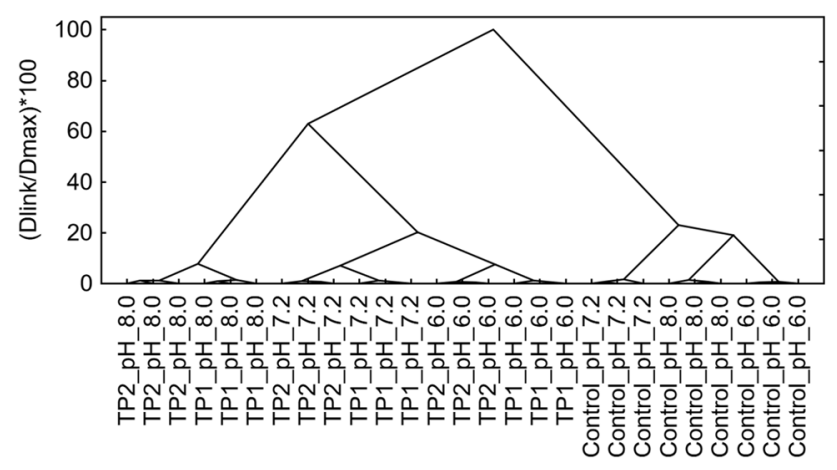

Fig. 1 Results of the cluster analysis showing the relation between tomato pomace (TP1 and TP2) parameters and variation of number of viable bacteria, concentration of short-chain fatty acids and concentration of bile acids at the three stages of digestion ( $\mathrm{pH}: 6.0,7.2$ and 8.0). The normalisation of scale tree to dlink/dmax $* 100$ was performed ( $\mathrm{d}$, distance; 1, linkage; max, maximum of linkage Euclidean distance). Amalgamation rule: Ward's method, distance metric: Euclidean distances found between the concentration of LHA, acetic acid, lactic acid, butyric acid, and the count of E. coli, Enterococcus spp., Lactobacillus spp. (Fig. 2A), while a negative correlation was found for Bifidobacterium spp.

This indicates low survival rate of Bifidobacterium spp. in experimental environment. The concentration of LHA was highest at $\mathrm{pH}$ 8.0. Bile acids appear to be a major regulator of the gut microbiota. Other authors demonstrated toxic activity of secondary bile acids on some lactic acid bacteria in previous research (Dziedzic et al., 2016; Merritt and Donaldson, 2009). Bile acids have both direct effect on fecal bacteria and indirect effect through induced antimicrobial peptides (Ridlon et al., 2014). Some authors described DCA as a much more antimicrobial agent compared to CA (Begley et al., 2005). Saito et al. documented that Enterococcus faecalis is able to use carbon source from fatty acids (Saito et al., 2014), therefore we suppose that fecal bacteria can utilize LHA, and its concentration is lowered in control sample in the absence of other bioavailable carbon source. On the other hand in TP samples the concentration of secondary bile acids increases, which can be linked to the role of some fecal bacteria in biotransformation of primary bile acids into secondary bile acids (Dziedzic et al., 2015). Comprehensive vector bundle consists of protein, propionic acid, DCA and CA variables. Analyzing the concentration of DCA and CA in control samples (Table 2) it can be concluded that these samples are featured by low solubility in acidic $\mathrm{pH}(6.2)$, and good solubility in basic environment ( $\mathrm{pH} 7.2$ ), where their concentration increased (Dziedzic et al., 2016). Higher solubility of these acids in control samples affected their bioavailability (the only carbon component) for fecal bacteria. As a result, at the final stage of digestion ( $\mathrm{pH}$ 8.0) a low concentration of these acids was observed (Table 2). Begley et al. (2005) described that E. coli growth was observed in the small intestinal compartments of digestive tract model in the presence of high concentrations of bile extract, whereas the content of Gram-positive bacteria decreased. Many authors suggested that Gram-positive bacteria are strain-specific taking into consideration tolerance to bile salts and their concentration (Chateau et al., 1994; Jacobsen et al., 1999; Zárate et al., 2000). Some evidence has shown that bile acids can be metabolized by some gut bacteria (Ruiz et al., 2013). The high concentration of these acids in TP samples does not only depend on the $\mathrm{pH}$ of the environment at individual stages of digestion, as confirmed by the location of vectors in Fig. 2A compared with samples in Fig. 2B. Proteins introduced together with pomace probably bond $\mathrm{CA}$ and DCA. These acids are negatively correlated with $E$. coli count, while remaining neutral to other analyzed bacteria. They also demonstrate positive correlation with propionic 
Table 3 K-means clustering, ANOVA results, evaluation of discriminant power of variables $(p<0.05)$; the final classification of control/tomato pomace (TP1 and TP2) samples at the three stages of digestion (pH: 6.0, 7.2 and 8.0)

\begin{tabular}{|c|c|c|c|c|c|c|c|c|c|}
\hline Variable & Between SS* & $\mathrm{df} *$ & Within SS* & df & $\mathrm{F}^{*}$ & $p$ value* & Case & Distance* & Final classification \\
\hline E. coli & 8.8313 & 2 & 17.1687 & 24 & 6.170 & 0.0069 & Control_pH_6.0 & 0.448 & 1 \\
\hline Enterococcus & 25.4231 & 2 & 0.5769 & 24 & 528.860 & 0.0000 & Control_pH_7.2 & 0.616 & 1 \\
\hline Lactobacillus & 22.1700 & 2 & 3.8300 & 24 & 69.460 & 0.0000 & Control_pH_8.0 & 0.608 & 1 \\
\hline Bifidobacterium & 17.8108 & 2 & 8.1892 & 24 & 26.100 & 0.0000 & TP1_pH_6.0 & 0.394 & 2 \\
\hline Lactic acid & 25.1193 & 2 & 0.8807 & 24 & 342.280 & 0.0000 & TP1_pH_6.0 & 0.366 & 2 \\
\hline Acetic acid & 25.2065 & 2 & 0.7935 & 24 & 381.210 & 0.0000 & TP1_pH_6.0 & 0.380 & 2 \\
\hline Propionic acid & 18.5188 & 2 & 7.4813 & 24 & 29.700 & 0.0000 & TP2_pH_6.0 & 0.318 & 2 \\
\hline Butyric acid & 25.3556 & 2 & 0.6444 & 24 & 472.140 & 0.0000 & TP2_pH_6.0 & 0.322 & 2 \\
\hline CA & 9.6018 & 2 & 16.3982 & 24 & 7.030 & 0.0040 & TP2_pH_6.0 & 0.330 & 2 \\
\hline DCA & 3.4941 & 2 & 22.5060 & 24 & 1.860 & 0.1770 & TP1_pH_7.2 & 0.337 & 2 \\
\hline LHA & 14.8327 & 2 & 11.1673 & 24 & 15.940 & 0.0000 & TP1_pH_7.2 & 0.338 & 2 \\
\hline Fat & 24.3406 & 2 & 1.6594 & 24 & 176.020 & 0.0000 & TP1_pH_7.2 & 0.309 & 2 \\
\hline Protein & 25.9633 & 2 & 0.0368 & 24 & 8478.050 & 0.0000 & TP2_pH_7.2 & 0.345 & 2 \\
\hline Ash & 25.9760 & 2 & 0.0240 & 24 & $12,964.190$ & 0.0000 & TP2_pH_7.2 & 0.394 & 2 \\
\hline NDF & 25.9003 & 2 & 0.0997 & 24 & 3118.380 & 0.0000 & TP2_pH_7.2 & 0.373 & 2 \\
\hline Cellulose & 25.2290 & 2 & 0.7710 & 24 & 392.650 & 0.0000 & TP1_pH_8.0 & 0.243 & 3 \\
\hline Hemicellulose & 25.2282 & 2 & 0.7718 & 24 & 392.240 & 0.0000 & TP1_pH_8.0 & 0.216 & 3 \\
\hline Lignin & 24.0645 & 2 & 1.9355 & 24 & 149.200 & 0.0000 & TP1_pH_8.0 & 0.220 & 3 \\
\hline SDF & 23.4196 & 2 & 2.5804 & 24 & 108.910 & 0.0000 & TP2_pH_8.0 & 0.224 & 3 \\
\hline IDF & 25.8610 & 2 & 0.1390 & 24 & 2233.130 & 0.0000 & TP2_pH_8.0 & 0.229 & 3 \\
\hline TDF & 25.8465 & 2 & 0.1536 & 24 & 2019.970 & 0.0000 & TP2_pH_8.0 & 0.226 & 3 \\
\hline
\end{tabular}

SS*, sum of squares between/within groups; df, degrees of freedom; $\mathrm{F}$, statistical F-test; $p$ value, probability value; distance, distance from respective cluster center; CA, cholic acid; DCA, deoxycholic acid; LHA, lithocholic acid; IDF, insoluble dietary fiber; NDF, neutral detergent fiber; SDF, soluble dietary fiber; TDF, total dietary fiber

Fig. 2 Tomato pomace samples (TP1 and TP2) at the three stages of digestion (pH: 6.0, 7.2 and 8.0) were presented in plot representing the PCA of loadings plot (A) and score plot (B); principal components-PC 1 and PC 2; CA, cholic acid; DCA, deoxycholic acid; LHA, lithocholic acid
(A)

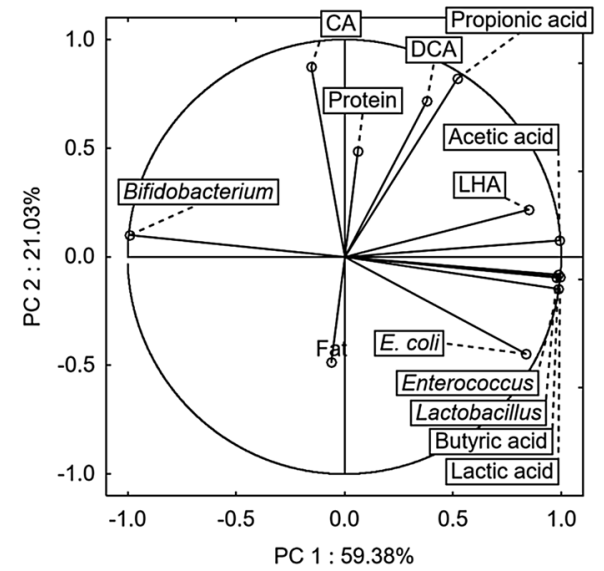

(B)

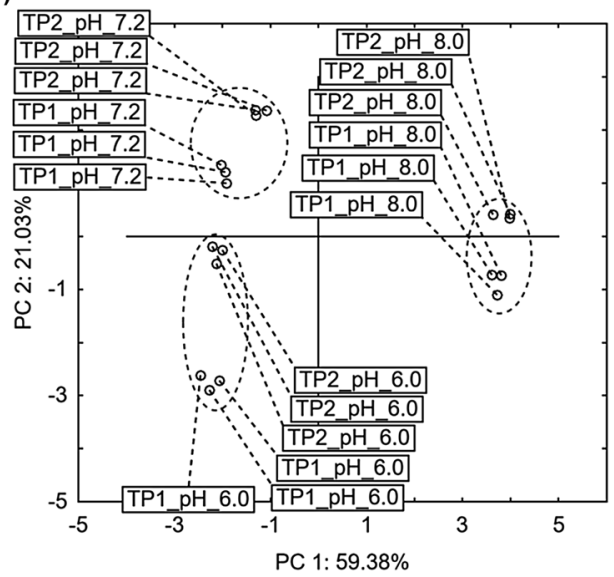

acid, which suggests a connection between the concentration of CA, DCA, and metabolites created by the bacteria.

It was found that fecal bacteria can use bile acids, i.e. CA and DCA, as their source of energy in an environment where carbon availability is limited. Furthermore, it was demonstrated that tomato pomace can significantly affect the count of fecal bacteria and the solubility of bile acids
(CA and DCA) in in vitro digestion due to bonding ability of their proteins/peptides. Obtained results prove that the availability and use of bile acids does not only depend on the interactions between bile acids and bacteria, but also the interactions of bile acids with other food components. The analysis of the influence of bacteria on bile acid concentrations in models without the food component does not 
reflect real life scenarios. An addition of tomato pomace to food can improve the condition of human digestive tract. However, the mechanism of bile acid sorption by tomato pomace, especially proteins and dietary fiber, and its resorption in digestive tract remains to be clarified. This would allow to conclude which of the considered factors have a significant effect on bile acid sorption.

Acknowledgements This research was supported by POIG Grant "New bioactive food with designed functional properties "POIG 01.01.02-00-061/09.

\section{Compliance with ethical standards}

Conflict of interest The authors declare that they have no conflict of interests.

Open Access This article is distributed under the terms of the Creative Commons Attribution 4.0 International License (http://crea tivecommons.org/licenses/by/4.0/), which permits unrestricted use, distribution, and reproduction in any medium, provided you give appropriate credit to the original author(s) and the source, provide a link to the Creative Commons license, and indicate if changes were made.

\section{References}

Bajerska J, Chmurzynska A, Mildner-Szkudlarz S, Drzymała-Czyż S. Effect of rye bread enriched with tomato pomace on fat absorption and lipid metabolism in rats fed a high-fat diet: nutritional effect of rye bread with tomato pomace in rats. J. Sci. Food Agric. 95: 1918-1924 (2015). https://doi.org/10.1002/jsfa. 6899

Begley M, Gahan CGM, Hill C. The interaction between bacteria and bile. FEMS Microbiol. Rev. 29: 625-651 (2005). https://doi.org/ 10.1016/j.femsre.2004.09.003

Böhm V. Lycopene and heart health. Mol. Nutr. Food Res. 56: 296-303 (2012). https://doi.org/10.1002/mnfr.769

Borguini RG, Ferraz Da Silva Torres EA. Tomatoes and tomato products as dietary sources of antioxidants. Food Rev. Int. 25: 313-325 (2009)

Chateau N, Deschamps AM, Sassi AH. Heterogeneity of bile salts resistance in the Lactobacillus isolates of a probiotic consortium. Lett. Appl. Microbiol. 18: 42-44 (1994)

Chen J, Song Y, Zhang L. Lycopene/tomato consumption and the risk of prostate cancer: a systematic review and meta-analysis of prospective studies. J. Nutr. Sci. Vitaminol. (Tokyo). 59: 213-223 (2013)

Dehghan-Shoar Z, Hardacre AK, Brennan CS. The physico-chemical characteristics of extruded snacks enriched with tomato lycopene. Food Chem. 123: 1117-1122 (2010)

Dehghan-Shoar Z, Mandimika T, Hardacre AK, Reynolds GW, Brennan CS. Lycopene bioaccessibility and starch digestibility for extruded snacks enriched with tomato derivatives. J. Agric. Food Chem. 59: 12047-12053 (2011)

Del Valle M, Cámara M, Torija M-E. Chemical characterization of tomato pomace. J. Sci. Food Agric. 86: 1232-1236 (2006)

Dziedzic K, Górecka D, Szwengiel A, Smoczyńska P, Czaczyk K, Komolka P. Binding of bile acids by pastry products containing bioactive substances during in vitro digestion. Food Funct. 6: 1011-1020 (2015)

Dziedzic K, Szwengiel A, Górecka D, Gujska E, Kaczkowska J, Drożdżyńska A, Walkowiak J. Effect of wheat dietary fiber particle size during digestion in vitro on bile acid, faecal bacteria and short-chain fatty acid content. Plant Foods Hum. Nutr. 71: 151-157 (2016)

Güneşer O, Demirkol A, Karagül Yüceer Y, Özmen Toğay S, İşleten Hoşoğlu M, Elibol M. Bioflavour production from tomato and pepper pomaces by Kluyveromyces marxianus and Debaryomyces hansenii. Bioprocess Biosyst. Eng. 38: 1143-1155 (2015)

Jacobsen CN, Rosenfeldt Nielsen V, Hayford AE, Møller PL, Michaelsen KF, Paerregaard A, Sandström B, Tvede M, Jakobsen M. Screening of probiotic activities of forty-seven strains of Lactobacillus spp. by in vitro techniques and evaluation of the colonization ability of five selected strains in humans. Appl. Environ. Microbiol. 65: 4949-4956 (1999)

Kun Y, Ssonko Lule U, Xiao-Lin D. Lycopene: its properties and relationship to human health. Food Rev. Int. 22: 309-333 (2006)

Merritt ME, Donaldson JR. Effect of bile salts on the DNA and membrane integrity of enteric bacteria. J. Med. Microbiol. 58: 1533-1541 (2009)

Previtera L, Fucci G, De Marco A, Romanucci V, Di Fabio G, Zarrelli A. Chemical and organoleptic characteristics of tomato purée enriched with lyophilized tomato pomace: characteristics of tomato purée enriched with lyophilized tomato pomace. J. Sci. Food Agric. 96: 1953-1958 (2016)

Primec M, Mičetić-Turk D, Langerholc T. Analysis of short-chain fatty acids in human feces: a scoping review. Anal. Biochem. 526: 9-21 (2017)

Reboul E, Borel P, Mikail C, Abou L, Charbonnier M, Caris-Veyrat C, Goupy P, Portugal H, Lairon D, Amiot M-J. Enrichment of tomato paste with $6 \%$ tomato peel increases lycopene and betacarotene bioavailability in men. J. Nutr. 135: 790-794 (2005)

Ridlon JM, Kang DJ, Hylemon PB, Bajaj JS. Bile acids and the gut microbiome. Curr. Opin. Gastroenterol. 30: 332-338 (2014)

Ruiz L, Margolles A, Sánchez B. Bile resistance mechanisms in Lactobacillus and Bifidobacterium. Front. Microbiol. 4: 396 (2013)

Saito HE, Harp JR, Fozo EM. Incorporation of exogenous fatty acids protects Enterococcus faecalis from membrane-damaging agents. Appl. Environ. Microbiol. 80: 6527-6538 (2014)

Shao D, Bartley GE, Yokoyama W, Pan Z, Zhang H, Zhang A. Plasma and hepatic cholesterol-lowering effects of tomato pomace, tomato seed oil and defatted tomato seed in hamsters fed with high-fat diets. Food Chem. 139: 589-596 (2013)

Stinco CM, Heredia FJ, Vicario IM, Meléndez-Martínez AJ. In vitro antioxidant capacity of tomato products: relationships with their lycopene, phytoene, phytofluene and alpha-tocopherol contents, evaluation of interactions and correlation with reflectance measurements. LWT Food Sci. Technol. 65: 718-724 (2016)

Ulleberg EK, Comi I, Holm H, Herud EB, Jacobsen M, Vegarud GE. Human gastrointestinal juices intended for use in in vitro digestion models. Food Dig. 2: 52-61 (2011)

Wohlsen T, Bates J, Vesey G, Robinson WA, Katouli M. Evaluation of the methods for enumerating coliform bacteria from water samples using precise reference standards. Lett. Appl. Microbiol. 42: 350-356 (2006)

Zárate $\mathrm{G}$, Gonzalez S, Chaia AP, Oliver G. Effect of bile on the $\beta$ galactosidase activity of dairy propionibacteria. Le Lait 80 : 267-276 (2000) 